\title{
Un prix et un hommage
}

ENGLISH VERSION ON PAGE 141

See related ARTICLE ON PAge 143

Bien que chaque édition du $7 C M U$ soit spéciale, celleci l'est particulièrement parce que nous y célébrons la soumission qui a remporté le prix annuel commémoratif de rédaction Penelope Gray-Allan du $7 C M U$. La gagnante du prix de cette année est Dre Michelle Tousignant, résidente au Programme de résidence en urgentologie du CMFC à l'Université de l'Alberta. Dre Tousignant a rédigé un essai très émouvant dont le sujet est poignant à tous égards, en raison de la perte tragique que le milieu canadien de l'urgentologie, l'Association canadienne des médecins d'urgence (ACMU), et le $7 C M U$ ont subie au début de 2010 par suite du décès prématuré de la rédactrice administrative du $\mathcal{F} C M U$ à l'époque, Penny Gray-Allan.

Le Prix commémoratif de rédaction Penelope GrayAllan du fCMU a été conjointement créé par le conseil de l'ACMU et le comité de rédaction du JCMU en guise de légitime hommage à Penny. Il honore la meilleure œuvre humanitaire réalisée par un résident canadien en urgentologie du CRMCC ou du CFMC. $\mathrm{Au}$-delà de la publication de leur œuvre, les gagnants du prix reçoivent les frais d'inscription, de déplacement et d'hébergement à la conférence annuelle de l'ACMU qui se tient cette année à St. John's, Terre-Neuve. Chaque année, les lecteurs du 7CMU peuvent désormais être heureux de lire l'œuvre gagnante dans l'édition de mai du journal.
Nous félicitons Dre Tousignant de sa remarquable publication et brillante réussite, et nous remercions les nombreux autres résidents qui ont soumis des articles pour ce concours, articles qui étaient tous pénétrants et émouvants. La qualité des soumissions de cette année inaugurale du nouveau prix augure bien pour le sangfroid, la perception et l'empathie de la nouvelle génération d'urgentologues canadiens. Nous remercions aussi les membres du comité de sélection à l'insu, Drs Jim Ducharme, Jeff Freeman et Peter Toth, pour l'expertise dont ils ont fait preuve dans le choix de la gagnante de cette année.

Les personnes qui ont travaillé avec Penny au comité de rédaction du $\mathcal{F} C M U$, ainsi que les nombreux auteurs et lecteurs critiques avec lesquels elle a interagi pendant ses années au $7 C M U$, se souviendront que pour autant qu'elle aimait la science et les propos précis, Penny appréciait également et par-dessus tout le côté humain de la médecine et les rapports étroits qu'elle avait établis dans le cadre de son travail. Penny, où que tu sois, nous espérons que l'article gagnant de cette année apportera un grand sourire à ton visage, comme ce fut le cas pour nous.

Riyad B. Abu-Laban, MD, MHSc

Corédacteur principal, JCMU

Chris Evans, MD

Président sortant, ACMU 